Final Paper- $20^{\text {th }}$ August 2009

\title{
Community Child Health (CCH) nurses' experience of home visits for new mothers: A quality improvement project
}

\begin{abstract}
This paper explores Community Child Health (CCH) nurses’ experience of home visits for new mothers in a health service region of Western Australia. Reported benefits of home visits include improvement in maternal and child health and better parenting skills leading to positive maternal-child interaction. Despite CCH nurses’ support for home visits factors such as costs, lack of resources and staffing issues have made home visits increasingly difficult. Twelve child health nurses participated in a focus group taped interview to explore how these nurses experienced home visits for new mothers in order to evaluate and improve home visits. Data were transcribed verbatim and content analysed. Three themes emerged from the nurses' experience of home visits. These were: 1) finding out about home visits with sub-themes such as scheduling first home visits and making the first phone call, 2) staying safe during home visits with sub-themes such as managing high risk clients and scheduling subsequent visits and 3) building positive partnerships with clients during home visits. The findings indicated CCH nurses followed a set pathway towards successfully completing home visits. This may provide valuable insights for other $\mathrm{CCH}$ nurses. Key words: Community nursing, home visits, child health nurses, new mothers.
\end{abstract}




\section{Introduction}

The topic of home visits for new mothers by community child health $(\mathrm{CCH})$ nurses was explored with a literature review. Since the last decade, home visits have noticeably increased with research highlighting the importance of the earliest years of a child's life and the benefits of home visits (Jansson, Petersson \& Uden 2001; Kemp et al. 2005; Olds \& Kitzman 1993; Olds et al. 1997; Plews et al. 2005). The literature (Kearney et al. 2000; Larner et al. 1992) shows that a common goal of CCH nurses is to improve the lives of children by encouraging changes in the attitudes, knowledge and or the behaviours of parents and caregivers. This is achieved by giving parents social support, practical assistance such as connecting them with appropriate community services, and education about effective parenting and child development (Kearney et al. 2000).

An exploratory Finnish study on home visits using 263 community nurses and 323 clients, found that clients equated home visits with professionals' assessing their baby for abnormalities and gaining information about the growth and development of their children. The nurses viewed home visits differently, as providing clients with the support and encouragement they needed to ensure continuity of care for their babies (Vehvilainen-Julkunen 1994). In another study on home visits conducted in England, Raynor (2001) reported increased parental involvement and reciprocal interaction, improvement towards and praise for the child, a more positive attitude towards the child and less difficulties with negative interactions. Moreover, Raynor indicated that home visits resulted in parents demonstrating realistic and positive expectations of their children with subsequent positive parenting (Raynor 2001). 
Further literature highlights the immense range of social problems that can be addressed by CCH nurses with home visits (McNaughton 2004). Whilst CCH nurses provide screening services to parents, there is now a trend towards a holistic approach to working with families with young children (Department of Health (DoH) WA, 2005). Other reported benefits of home visits include improvement in maternal and child health, better parenting skills leading to positive maternal-child interaction, and positive outcomes for the family (Kearney et al. 2000). Home visits allow CCH nurses to discuss preventative strategies with individual families and to explain how to navigate through the various government systems to access much needed resources (Kemp et al. 2005).

McNaughton (2000) reported on a synthesis of available qualitative research on maternal home visits from England, United States, Canada and Finland and discussed the many reports confirming the benefits of home visits to clients. McNaughton (2000) concluded that building a positive relationship with clients formed the basis of problem identification and problem solving leading to client empowerment (McNaughton 2000: 405). Additionally, McNaughton indicated that further research was needed to examine how home visits were conducted and the impact on client outcomes as well as how demographic family factors influence the number of home visits. Further she reported that one home visit did not yield as positive an outcome for the client as two or more visits (McNaughton 2000).

McNaughton (2000) also asks 'what happens over time to produce changes in the client's behaviour and what does current research report about the processes involved in home visits?' (McNaughton, 2000: 407). Barnes et al's (2003) focus group study of 
22 Queensland CCH nurses provides information on current nursing practice and challenges. The most significant challenge identified was concerning the limited resources available and the inference that there were more at risk families than resources available for these families. This may impact on the families without risk factors who may develop problems as a result of reduced home visits by $\mathrm{CCH}$ nurses.

\section{The Australian Context}

The length of postnatal hospital stays in Australia has decreased. This may mean new mothers’ need for greater support and education from CCH nurses after discharge. Child health nurses are in a unique position to work with families to promote health and well being, applying the research evidence about the importance of early childhood also termed as 'best beginnings' (Kemp et al. 2005: 255). Child health nurses are well respected in the community and are accepted by parents as they are viewed as not making judgements or stigmatising families (Downie et al. 2004). There is evidence to indicate that families play a vital role in the way children grow and develop (Kearney et al. 2000). There is literature to suggest that effective health interventions by CCN nurses working in partnership with families at key points in a child's life can have a positive impact on the child's developmental trajectory (Kemp et al. 2005). The thrust of the partnership is recognition of the mother's and family's expertise rather than the nurse being the 'expert' (Kemp et al. 2005: 258). Through partnership there is building of trust, rapport, and genuine interest by both parties. There is ongoing provision by the nurse of much needed information and support with regards to child development and family situations, advocacy, and addressing the immense range of social problems often facing families (McNaughton 2004). The 
optimum way CCH nurses can achieve the above outcomes is through home visits, especially in the first 10 days after the birth of the child.

\section{Home visits in Western Australia}

The focus of home visits is to establish a relationship between the parents/caregivers and the $\mathrm{CCH}$ nurse in a setting where the family feels safe and in control. The first visit is not prescriptive but is intended to facilitate the building of a trusting relationship between the parent/ family enabling the nurse to provide effective services to families in an acceptable manner. During the home visit the nurse conducts a social assessment for the purpose of identifying family resources, assessing the environment in the home and any issues parents may have (DoH 2005). The nurse uses the risk and protective factor guide (DoH 2005) to assess for high risk factors such as domestic violence in the family so as to make decisions regarding planning the appropriate action. If risks are identified some examples of actions may be to do a follow up second visit, make referral to other relevant allied health services, or to provide additional monitoring and services (DoH 2005). During the initial visit, the $\mathrm{CCH}$ nurse discusses and addresses issues such as infant feeding and associated aspects like feeding difficulties, proper attachment to breast, expressing breast milk, and formula feeding. The nurse also discusses child development, maternal physical and emotional well being, injury prevention and safety, child care, and immunisation schedules (DoH, Child Health Record 2005). The CCH nurse uses a holistic approach when conducting home visits. In order to achieve this holistic approach to service provision, $\mathrm{CCH}$ nurses have had to change the manner and the process by which they conduct home visits (DoH WA 2005). 


\section{Method}

A descriptive qualitative method using focus group interview as a data collection strategy was used to evaluate home visits. The qualitative method facilitated the gaining of insight into the social reality of $\mathrm{CCH}$ nurses in the focus group, that is, their thoughts and feelings about how they perceived the process of home visits (Morgan 1997). Focus group interview was chosen to conduct this evaluative project as it is a type of self contained method that draws out meanings that people attach to their experiences of home visits. The focus group interview presented a more natural environment than that of an individual interview for the $\mathrm{CCH}$ nurses to influence and be influenced by others in the group, just as people are in life (Krueger 1994). Furthermore, the focus group interview facilitated comparisons to be made between perceived experiences of the group's participant as well as allow the researcher to gather more data in less time (Morgan 1997). The number of home visits CCH nurses conducted and their opinions about the outcomes of the visits were also explored in the focus group interview.

\section{Approval to conduct the focus group interview}

Approval to conduct the focus group interview was obtained from the Program Manager of Community Health of the selected health service region in Western Australia. As this was a quality improvement project, the researcher was assured that formal ethical approval was not required by management. Permission to access the sample was obtained from the Program Manager of Community Health of the selected health service region in Western Australia. Participants were approached at a routine meeting by the researcher to request their involvement. Consent was ensured by 
seeking permission to tape the interview. Permission was obtained from the participants for a scribe to be present during the interview. Participants were given a brief overview of the project and the protocol for the focus group was explained. They were assured that all information collected will be coded and only de-identified data will be presented in the final report. As well, assurance was given that they could request for the tape recorder to be turned off at any time during the interview or withdraw their participation without penalty. The participants were informed that written consent will be obtained from them prior to commencement of the interview. They were assured that following transcription of the tape and verification by them for accuracy, the tapes will be erased to avoid voice identification. All hard copies of the transcription will be kept in a locked secure cupboard.

\section{Data Collection}

Twelve community child health nurses agreed to participate in the focus group interview. The participants had nine to seventeen years of home visiting experience. The interview was held at a time and place that suited participants. Open-ended semistructured questions were asked with the researcher using probing questions to seek further clarification on responses given by participants. The participants were asked questions such as, 'what has been your experience with home visits for new mothers? 'How do you prepare for your visit?' 'Can you share with me what happens during the home visit?' 'What are your thoughts on high risk clients?' Discussion also occurred during the focus group interview which allowed some participants to examine ideas not considered before and others to give further information to clarify statements. The interview lasted one hour, followed by a short debriefing session after the tape was turned off. The scribe took notes during the interview. At the end of the 
interview key points were read back to the participants for consensus regarding the content of the interview. Three of the participants were later contacted by telephone to further expand on comments they had made during the interview.

\section{Data Analysis}

The interview was transcribed verbatim. Once transcribed the data were analysed by coding, finding themes and clustering (Struebert \& Carpenter 1999). The data were examined line by line and significant words and phrases were identified. Following this, the major intent of the data was conceptualised (Field \& Morse 1992). The next step was clustering these concepts and comparing them again with each other to ensure that they were mutually exclusive. A final list of themes was developed.

\section{Findings}

Data analysis centred round three themes which described CCH nurses' experience of home visits for new mothers. These were:

1. Finding out about home visits with sub-themes such as scheduling first home visits and making the first phone call.

2. Staying safe during home visits with sub-themes such as managing high risk clients and scheduling subsequent visits.

3. Building positive partnerships with clients during home visits.

Although these themes appear to be discreet items, it is important to understand that there was merging of the themes and sub-themes when taken into the context of home visits. For example, the theme of staying safe was strongly linked with the sub-theme of making the first phone call. Moreover, the findings themselves are not placed in 
any order of importance but in such a way as to permit the data to speak for itself which is characteristic of qualitative analysis. The three themes and sub-themes are described in the following section.

\section{Theme 1: Finding out about home visits}

The theme of finding out about, which mothers needed home visits, was considered to be the first step in conducting home visits. Once the nurses found out they planned and scheduled the first visit and made the first phone call. These are the sub-themes. The nurses in the focus group reported that the greatest difficulty for them was finding out about new mothers needing home visits. The manner in which the nurses gained this information was varied and often delayed leaving the nurses frustrated and unsure. For example, analysis of the data revealed that the nurses generally became aware of new births in their area through several sources and that there was no standard means by which they were notified about a visit. The nurses stated they might receive a birth notification via land mail or receive a phone call from another child health nurse. They could also be contacted by a midwife from the hospital where the mother was discharged and had expressed health concerns for the baby and or mother such as illness, high need or risk of maternal depression. Occasionally the nurses were notified by the Department of Community Development (DCD) about high-risk clients. This is highlighted by this comment:

We receive a birth notification via the clinic for the new baby or we contact the main office ... we may get a phone call from another nurse to say the client has moved to our area. A hospital source may contact to say they have concerns about a client...DCD may ring and make us aware that the client has had a baby and that the baby may be at risk and ask us to follow up. 
Some nurses expressed concern over the timeliness of when birth notices reached them. For example, some nurses did not receive the notices until 5-10 days after the mother and baby were discharged. There was also the issue of mail being unreliable especially in certain areas with some notices arriving when the baby was 3 weeks old or on a few occasions not arriving at all. The nurses stated electronic birth notices arrived on time but did not contain sufficient information or had insufficient details. Others claimed the antenatal details were sometimes not updated at birth. For example:

Very variable [birth notices] in our area, could be five days, very unreliable ... we get clumps of them and then a bit of a lull and they [babies] can be three weeks old by the time we get them ... we can also get the wrong address and we have to wait for the client to contact us ...this can be frustrating!.

The nurses also gave other reasons for not conducting home visits within the first 10 days after birth. They explained:

Mothers in private hospitals do not always wish for us to do a home visit as they had stayed in hospital for 7, 8, or 9 days. Also it can be difficult to track teenage mothers down. Sometimes new mothers' lack of knowledge in how to contact us or even understanding our role can be a problem. Some think that because they [mothers] have had a home birth they may not need a home visit.

These above response from the nurses indicate there may be client related reasons of why home visits may not be possible beyond getting timely birth notices. 


\section{Sub-theme-Scheduling first home visits}

Once the nurses found out about which clients needed home visits they made every effort to schedule the first visit. Nearly all the nurses stated they put aside time for home visits in their daily routine although this was not consistent. For some, it was a matter of filling in the gaps between seeing clients in the clinic and doing the visits. It was evident from nurses' statements that they used prior knowledge to gauge how many clients they are likely to have in the morning and try and fit in visits in the afternoon. There seemed to be no standard method for scheduling visits as highlighted by this comment by one nurse:

Again it is when you have got the gaps and I find that I try to dedicate a certain amount of time to see clients ... I leave gaps hoping that they [clients] will ring.

Another nurse added:

We actually have a system of where we mark off time for visits and allow how much time because we have got a rough idea how many births we have on an average so we allow that amount of time, in the diary ahead of time.

\section{Sub-theme: Making the first phone call}

The sub-theme of making the first phone call was considered to be vital by the nurses. This was because once the nurses found out about the first visit they needed to gain information about the client they were about to visit for the first time. According to the nurses the information they were seeking through the phone call often assisted them to decide on what they needed to do prior to the visit. Most nurses were of the 
opinion that the first phone call had many benefits to themselves and to the client as highlighted by this statement:

We introduce ourselves to the client ... assess how quickly we need to see the client, for example, in the case of post natal depression. We basically get a relationship going with the client ... we give them contact numbers of community resources that they may need immediately. Through talking with the client we gain information about the mother and baby that we think may be missing in birth notices.

The nurses reported that the first phone call took anywhere between 1-10 minutes. They also perceived making this call to be an essential part of the nurse's role in building a relationship with the mother prior to the actual visit as a precursor to working in partnership with the mother. The nurses stated that it was good for the mother to have the same person for the phone call and visit. Some mothers were also perceived to be unaware of home visits in which case the first phone call enabled the nurse to provide information, check for correct addresses for the visit, identify immediate needs the mother may have, and make an appointment for the visit. The nurses also claimed the first phone call allowed for active listening to pick up on any underlying negative factors impacting on the mother and baby as reflected by this nurse’s statement.

When you make the phone call you are really talking with the mum and assessing what is happening between mum and baby over the phone and also prioritising your workload within the clinic ... listening over the phone to hear any warning signs that things are not going well. 


\section{Theme 2: Staying safe during home visits}

The second theme identified was 'Staying safe during home visits'. It was further divided into two sub-themes 'Managing high risk clients' and 'Scheduling subsequent home visits’. Most times the nurses knew that their client was high risk and accordingly they took all the necessary precautions including using their skills in managing high risk clients. This sub-theme is described in the following section.

\section{Sub-theme: Managing high risk clients}

The nurses expressed concern that sometimes they did not have all the information about clients and this status had been unsettling for them. For example, the nurses indicated they were not always aware of factors that may confront them during home visits especially with high risk clients such as:

We don't always pick up clues about clients being high risk during the first phone call so you can be really going into homes 'blind' ... it can be unnerving at times ... most common risk is domestic violence ...that virtually comes from social issues and you really have to think about that if you are going in unawares of the dangers.

Accordingly, the nurses took various precautions to safeguard themselves.

We have got a policy about risk management that we take our mobile phones to the home visit and make sure someone knows where you have gone. There is a policy about training to get out of difficult situation, how to assess if things are escalating. How to get out! 
The nurses used information they gleaned from the first phone call and intuition to gauge risks and to assess risks when visiting high risk clients. For example:

Sometimes when I make a phone call I can hear things going on in the background that might alert you to the fact that it might not be a safe place to visit by yourself. I have rung people up and heard angry people in the background, swearing and cursing about someone ringing up and that sort of thing and for that client I have taken another nurse with me when I do the home visit.

Other times though you get a sixth sense that something is not quite right in the home ... it is like you know there is going to be trouble ... 9 out of 10 times you are right.

Sometimes the maternity hospital will let them know if there was a risk but not always. A few of the nurses explained various strategies that they used to minimise risks pre and during visits such as:

We listen for background noise such as 'yelling', 'swearing' 'shouting' 'dog barking' during $1^{\text {st }}$ phone call to the new mother. We receive official notice about drug users and we act on 'gut feeling' and not go into the client's house. Ask for the dog to be restrained before entering the house for example ... 
We use our mobile phone to check who is in the house before entering ... we often will ring from the car. We will park the car in a strategic direction so we can drive off quickly without having to reverse ... we always follow protocol as set out by the Department of Health. Show the client that we are non-judgemental and only interested in the welfare of the child by only having eye contact with the mother and baby.

Furthermore, some nurses stated they were generally accepted in the homes because they were not viewed as a threat by clients. They were confident in their skills for defusing difficult situations that may arise during visits as highlighted by these statements.

I think nurses are accepted in the homes, we are not a threat to them [clients] and I think we are really good at defusing things if things appear to be a bit uptight when we go there ... I think the nurse generally has the skills to defuse it and to get them [clients] to focus on the baby.

Another nurse stated:

I have actually been on a visit when both parents were blind drunk to see a new baby and I went in with the door between me and them [parents] and although they were blind drunk they could see that I was concerned about the baby.

An example of how one nurse reduced her risk in a difficult home situation was to encourage the mother to come to the clinic for subsequent visits and to reassure her that she can get support at the clinic as indicated by this comment. 
There have been some agro men around in the background, but I just focus on the mother and the baby and try and ensure there is safety and make an appointment for her [mother] to come to the clinic next time ... ensure that the mother knows that she does have an escape route.

All the nurses were of the view that home visits should be done by two nurses for safety reasons however this was not always possible. They suggested working in groups in the clinics so there are enough nurses to run the clinic as well as conducting home visits safely. This is highlighted by this statement:

Child health nurses should work in teams ...this will facilitate nurses to go out in pairs if required to reduce personal risks to nurses and the nurses not going out on visits are able to run the clinic uninterrupted.

\section{Sub-theme: Scheduling subsequent home visits}

The nurses explained they offered subsequent home visits if they perceived risk factors. These included: the presence of domestic violence, evidence of drug use in the parents, if the mother was a teenager, if the mother was a sole parent, if there was evidence of postnatal depression and or mental illness, concern for the baby's or mother's health or if the mother was experiencing breast feeding difficulties. One nurse stated:

If you pick up from what you have been talking about or what you have seen in the home ... sole parent, drug use, domestic violence, lower socio-economic 
issues. These are regular now. So you are looking at these things and listening to mum when you are there, if she [mother] has a history of depression, is having problems with breast feeding or feeling tired and baby not settling, I often ask them 'would you like to be seen again? Some will say yes, and some will say no.

It was reported by some nurses that all mothers who called and asked for a second visit received one. The number of second or subsequent visits varied amongst areas. For example one nurse stated that she often offered a second home visit to mothers who were not perceived to be high risk but for social reasons such as the inability to come to the clinic. The nurse commented:

It's kind of a social issues, not necessarily a high risk ... maybe the mother has 6 children or something like that ... because they [mothers] cannot come to the clinic'. Other times it may be lack of transport for the mother or cultural reasons, quite a lot of people only have one car, I am finding that a lot more.

In subsequent visits the nurses spent less than an hour with clients as they have already established a rapport and relationship with them as highlighted by this comment:

Can be quite quick as we have established a good relationship ...you are really picking up from where you left off the last time ... I don't mind second visits as it can be quite quick. 


\section{Theme 3: Building positive partnerships with clients during home visits}

The nurses perceived that their current practice involved working in partnership with families that included parents, babies, siblings and extended family. According to the nurses the key thrust of the visit is to build a positive partnership with the client which they perceived to be crucial in working effectively with them. They stated:

It's building up partnerships isn't it? And a healthy relationship really and then that comes to health promotion and education and doing the linking with counselling, advocacy, social workers ... addressing the mental and physical health of mother and baby.

One strategy the nurses stated they used to build the positive partnership was to engage with the mother in a general sense initially such as asking about the physical and mental well being of the mother and baby. For example, the nurses stated they started with delivery details and conducted a needs assessment. They asked about breast feeding including checking for appropriate attachment techniques, nutrition, sleep pattern, medical details, social support needed, and emotional well being as reflected by these statements:

In building partnership I usually start with medical history ... talking about the delivery is a good ice breaker ... helps with building rapport and trust. Ask about family support, support in the community, birth history, post natal problems, partner or no partner, emotional well being, nutrition, contraception, and mother's health. 
Another nurse reported:

You have a mental plan I suppose of the areas that you need to cover but by the same token you are going in there and you need to be focussed on what the client wants to discuss and what their needs are at the time ... it is building a partnership isn't it ... the mother's mental health and her relationship with the baby, whether she needs support. So things like the psycho-social aspect. Some are just routine standard and then there is every deviation of the normal format ... I think you have to work with them [client].

\section{Discussion}

This evaluative project explored how a small sample of CCH nurses experienced home visits. Three themes were identified in the project. These were 'Finding out about home visits’, ‘Staying safe during home visits’, and ‘Building positive partnership with clients during home visits'. From the findings it may be inferred that the nurses preferred the fast electronic method of knowing about birth notices as long as the notices were complete and with sufficient and updated information. By receiving timely birth notices the nurses were able to visit within the first 10 days of the child's birth. Making the first phone call before the visit was also important for nurses from the findings. From their statements, it may be inferred that the nurses perceived themselves to be the best person to make the first phone call. Through this call, nurses were able to quickly assess the clients' situation, offer immediate help and prioritise the visit. The first contact also established the building of a relationship between the nurse and client. Hence, the nurses were adamant that the first phone call should be part of the nurses’ role. 
As for 'Staying safe during visits, the Department of Health in Western Australia has a security policy for Home Visiting Nurse Services (DoH, 2005). In the policy nurses are expected to assess risks pre and during home visits. Clients are either categorised as low risk whereby there is no indication of violence and or aggression. Clients are classed as high risk if there is evidence of aggression and or violence, substance abuse, restraining order, custody issues etc. The nurses conducting home visits also undergo staff training in managing aggression and or risky situations (DoH, 2006). The nurses in this evaluative project all indicated that they were cognisant of the policy and felt competent in getting out safely in difficult situations. Their main goal was to ensure personal safety to themselves and the infant and mother. To this end the nurses employed various strategies to conduct a safe visit as per the findings.

From the findings, the nurses' reliance on establishing partnerships with the mother/caregiver provided the basis upon which the nurses conducted home visits and provided care. Jansson et al.'s (2001) paper on nurses' encounter with parents with newborn babies flagged the importance of sound relationships between parents and nurses. These authors reported that it is only through such a relationship that nurses can work effectively with parents/caregivers. In this evaluative project, the nurses engaged with the mothers by addressing general physical and psychosocial issues first as an ice breaker towards building rapport and trust and eventually a partnership. The perceived benefits of home visits as highlighted by participants in this study concur with Olds and Kitzman's (1993) report on how home visits improved the lives of children through providing social and practical support to parents. The child health nurses in this project reported they actively promoted health education and promotion 
especially in the area of nutrition, effective parenting and normal growth and development of children. This is supported by Kemp et al.(2005) who suggested that parents who have knowledge and support are assisted in changing attitudes and becoming better parents. Studies by Downie et al. (2004), McNaughton (2000), Raynor (2001), Vehvilainen-Julkunen (1994), also bear resemblance to the findings. McNaughton particularly challenged the value of one to two home visits to mothers and families. In this project, only two visits were accomplished by CCH nurses.

\section{Implications}

The main implications for $\mathrm{CCH}$ nurses from this evaluative project are the following:

- The need for a standard means of birth notification in a timely manner so home visits can be conducted within the first 10 day of birth. An electronic method with updated relevant information may need to be considered.

- There needs to be a standard method for scheduling home visits rather than ad hoc scheduling when $\mathrm{CCH}$ nurses can find a gap in their busy clinic hours. Ensuring a standard schedule may assist with formal time being made available for home visits.

- Community Child Health nurses may need to consider working in teams in clinics to facilitate 2 nurses going out together for home visits to reduce personal risks. At the same time the nurses not doing home visits can run the clinic uninterrupted.

\section{Limitations}

The findings of this project can only be considered in the context of the setting in which data was gathered. A limitation of the findings is the small sample size of 12 in 
a single focus group. There is also the possibility that the nurses in the focus group self-selected to be in the interview. This may explain the one-sided view of home visits, and that is, support for home visits. It would have been interesting to obtain the views of other nurses who may present a different view. Future research into $\mathrm{CCH}$ nurses’ experiences of home visits needs to include more than one focus group and at different sites. The findings of this project, however, do give an insight into the value of home visits and how other $\mathrm{CCH}$ nurses can improve the process of home visits by reading about the experiences of these 12 nurses.

\section{Conclusion}

Community child health nurses recognise the importance of the early years of life and that child development and parenting all need special support (Marshall \& Craft 2000; Stanley 2003). Whilst the nurses in this evaluative project were very supportive of home visits they also highlighted some shortcomings with home visits that can be addressed at the local level. Nevertheless, the nurses offer useful strategies that other $\mathrm{CCH}$ nurses can use in other similar context. Of particular use is the manner in which the nurses in this project ensured their personal safety with high risk clients.

\section{Acknowledgements}

The author is grateful to all the child health nurses who gave up their valuable time to be in the focus group interview and for willingly sharing their experiences of home visits. Without their support this project would not have been possible. Thanks also goes to Ms Selena Knowles who kindly gave her time in scribing key points during the interview and for reading the final draft of the report and providing feedback. A special thanks to Ms Angela Poole for facilitating access to the sample. 


\section{References}

Barnes M, Courtney M, Pratt, J and Walsh A (2003) Contemporary child health nursing practice: Services provided and challenges faced in metropolitan and outer Brisbane areas. Collegian 10:14-19.

Department of Health WA (DoH) (2005) Birth to school entry: Universal prevention and early detection schedule, policy rationale and summary of the changes to the schedule. Department of Health: Child Health Record (2005).

Downie J, Hubble J, Munns A and Wynaden D (2004) Changing focus of practice for community health nurses: Advancing the practice role. Contemporary Nurse 16: 208-213.

Field PA and Morse JM (1992) Nursing research: The application of qualitative Approaches, Chapman \& Hall, London.

Jansson A, Petersson K and Uden G (2001) Nurses’ first encounters with parents of new-born children: Public health nurses' views of a good meeting. Journal of Clinical Nursing 10:140-160.

Kemp L, Anderson T, Travaglia J and Harris E (2005) Sustained nurse home visiting in early childhood: Exploring Australian nursing competencies. Public Health Nursing 22: 254-264.

Kearney MH, York R and Deatrick JA (2000) Effects of home visits to vulnerable young families. Clinical Scholarship 32: 369-376.

Krueger RA (1994) Focus groups: A practical guide for applied research $2^{\text {nd }}$ edn, Sage Publications, Thousand Oaks.

Larner M, Halpern R \& Harkavy O (1992) Fair start to children: Lessons learned from seven demonstration projects, New Haven, Connecticut, Yale University Press. 
Marshall J and Craft K (2000) New vision for community health services for the future report, Perth, WA, Department of Health WA.

McNaughton DB (2004) Nurse home visits to maternal-child clients: A review of intervention research. Public Health Nursing 21: 207-224.

McNaughton DB (2000) A synthesis of qualitative home visiting research. Public Health Nursing 17: 405-414.

Morgan DL (1997) Focus groups as qualitative research $2^{\text {nd }}$ edn, Sage Publications, Thousand Oaks.

Olds, DL and Kitzman H (1993) Review of research on home visiting for pregnant women and parents of young children. The future of Children 3: 53-92.

Olds DL, Eckenrode J, Henderson CR, Kitzman H, Powers,J and Cole R (1997) Long term effects of home visitation on maternal life course and child abuse and neglect-fifteen year follow up of a randomised trial. JAMA: The Journal of the American Medical Association 278: 637-643.

Plews C, Bryar R \& Closs J (2005) Clients’ perceptions of support received from health visitors during home visits. Journal of Clinical Nursing 14:789- 797.

Raynor P (2001) Review: Home visiting programmes that include greater than or equal to postnatal home visits are associated with improved quality of home environment and parenting. Evidence-based Nursing 4: 9.

Streubert HJ and Carpenter DR (1999) Qualitative research in nursing: Advancing the humanistic imperative ( $2^{\text {nd }}$ edn), Philadelphia, Lippincott.

Stanley F (2003) Why putting children first is so important. National press club address. Available at http://www.chnwa.org.au/files/press_club_address.pdf. accessed 20th July 2008 
Vehvilainen-Julkunen K (1994) The function of home visits in maternal and child welfare as evaluated by service providers and users. Journal of Advanced Nursing 20: 672-678. 